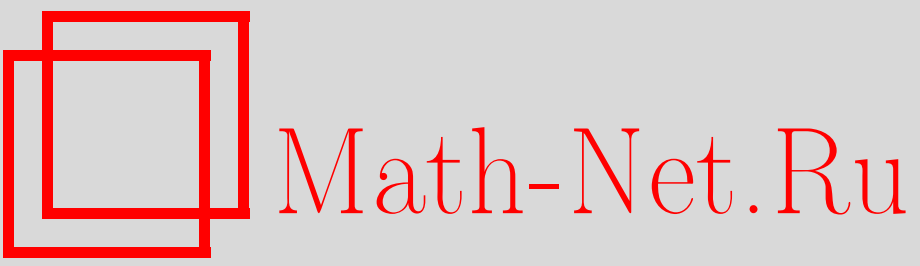

М. И. Бесова, Голоморфная регуляризация в теории краевых задач, Итоги науки и техн. Сер. Соврем. мат. и ее прил. Темат. обз., 2021, том 193, 11-16

DOI: https://doi.org/10.36535/0233-6723-2021-193-11-16

Использование Общероссийского математического портала Math-Net.Ru подразумевает, что вы прочитали и согласны с пользовательским соглашением

http://www.mathnet.ru/rus/agreement

Параметры загрузки:

IP : 54.157 .27 .8

26 апреля 2023 г., 14:12:53 


\title{
ГОЛОМОРФНАЯ РЕГУЛЯРИЗАЦИЯ В ТЕОРИИ КРАЕВЫХ ЗАДАЧ
}

\author{
(c) 2021 г. $\quad$ М. И. БECOBA
}

\begin{abstract}
АннотАция. Метод голоморфной регуляризации сингулярных возмущений применен для изучения краевой задачи для уравнения второго порядка. Доказано, что после точного описания сингулярностей регулярная часть решения аналитически зависит от параметра.
\end{abstract}

Ключевые слова: тихоновская система, голоморфная регуляризация, аналитический интеграл, псевдоголоморфное решение.

\section{HOLOMORPHIC REGULARIZATION IN THE THEORY OF BOUNDARY-VALUE PROBLEMS}

\section{(C) 2021 M. I. BESOVA}

\begin{abstract}
The method of holomorphic regularization of singular perturbations is applied to a boundary-value problem for a second-order equation. We prove that after an precise description of singularities, the regular part of the solution depends analytically on the parameter.
\end{abstract}

Keywords and phrases: Tikhonov system, holomorphic regularization, analytic integral, pseudoholomorphic solution.

AMS Subject Classification: $34 \mathrm{~B} 15$

1. Введение. В настоящее время для решения краевых сингулярно возмущенных задач наиболее широко используется метод погранфункций Васильевой-Бутузова-Нефедова (см. [1]). Для доказательства существования решения на всем промежутке задания уравнения в нем применяется метод дифференциальных неравенств, в основе которого заложены идеи Чаплыгина и Нагумо $[2,9]$ о верхних и нижних решениях. Для построения решения краевой задачи используется «метод стрельбы», законность применения которого фактически означает существование решения соответствующей начальной задачи на всем промежутке. В данной работе изложен подход, связанный с голоморфной регуляризацией сингулярно возмущенных задач $[3,4]$. Этот подход является продолжением идей С. А. Ломова о существовании, при определенных условиях, решений, представимых сходящимися в обычном смысле рядами по степеням малого параметра [6-8]. Такие решения называются псевдоаналитическими (псевдоголоморфными) и ранее строились преимущественно для начальных задач, причем локально [3-5], поэтому в работе применяется алгоритм псевдоголоморфного продолжения решений.

2. Метод голоморфной регуляризации и аналитические по параметру интегралы. Исследуем на отрезке $[0,1]$ следующую краевую задачу:

$$
\begin{gathered}
\varepsilon y^{\prime \prime}=f\left(x, y, y^{\prime}\right), \quad 0<x<1, \\
a_{1} y(0)-a_{2} y^{\prime}(0)=a, \quad b_{1} y(1)+b_{2} y^{\prime}(1)=b, \\
a_{2} \geqslant 0, \quad b_{2} \geqslant 0, \quad a_{1}^{2}+a_{2}^{2}>0, \quad b_{1}^{2}+b_{2}^{2}>0
\end{gathered}
$$


с малым положительным параметром $\varepsilon$. Сведем задачу (1) к краевой задаче для тихоновской системы $[1,5]$ с одной быстрой и одной медленной переменной:

$$
\begin{gathered}
\left\{\begin{array}{l}
y^{\prime}=v, \\
\varepsilon v^{\prime}=f(x, y, v),
\end{array}\right. \\
a_{1} y(0)-a_{2} v(0)=a, b_{1} y(1)+b_{2} v(1)=b .
\end{gathered}
$$

В соответствии с методом голоморфной регуляризации, перейдем от нелинейной системы к линейному уравнению ее интегралов [3,5]:

$$
\varepsilon L U+f(x, y, v) U_{v}=0,
$$

где $L=\partial_{x}+v \partial_{y}$ - дифференциальный оператор первого порядка в частных производных. Будем считать оператор $L$ подчиненным оператору $f \partial_{v}$, и в этом случае решение уравнения (3) ищем в виде регулярного ряда по степеням малого параметра:

$$
U(x, y, v, \varepsilon)=U_{0}(x, y, v)+\varepsilon U_{1}(x, y, v)+\ldots+\varepsilon^{n} U_{n}(x, y, v)+\ldots
$$

Применив метод неопределенных коэффициентов, получаем следующую серию задач:

$$
\begin{aligned}
\left(f \partial_{v}\right) U_{0} & =0 \\
\left(f \partial_{v}\right) U_{1} & =-L U_{0} \\
\left(f \partial_{v}\right) U_{2} & =-L U_{1} \\
& \cdots \\
\left(f \partial_{v}\right) U_{n} & =-L U_{n-1}
\end{aligned}
$$

Зададим для функции $f(x, y, v)$ условия $(\alpha): f(x, y, v)$ аналитична на ограниченной замкнутой области $\bar{\Omega}_{x y v} \subset \mathbb{R}^{3}, f(x, y, v) \neq 0 \forall(x, y, v) \in \Omega_{x y v}$ и обращается в нуль на ее границе $\Lambda$. Предположим также, что отрезок $[0,1]$ принадлежит $\bar{\Omega}_{x y v}$ и ее проекции $\bar{\omega}_{x y}$ на пространство $\mathbb{R}^{2}$. В качестве решения первого уравнения серии (5) рассмотрим произвольную функцию $\psi(x, y)$, аналитическую на замкнутой области $\overline{\omega_{x y}}$. Обозначим через $\widetilde{v}$ значение быстрой переменной $w$ на левом конце отрезка задания уравнения и потребуем, чтобы $U_{n}(x, y, \widetilde{v})=0, n=1,2, \ldots$ В результате имеем:

$$
\begin{aligned}
& U_{1}(x, y, v)=-\int_{\widetilde{v}}^{v} \frac{L_{1} \psi d v_{1}}{f\left(x, y, v_{1}\right)}, \quad L_{1}=\partial_{x}+v_{1} \partial_{y} ; \\
& U_{2}(x, y, v)=\int_{\widetilde{v}}^{w}\left[L_{1} \int_{\widetilde{v}}^{v_{1}} \frac{L_{2} \psi d v_{2}}{f\left(x, y, v_{2}\right)}\right] \frac{d v_{1}}{f\left(x, y, v_{1}\right)}, \quad L_{2}=\partial_{x}+v_{2} \partial_{y} ; \\
& U_{3}(x, y, v)=-\int_{\widetilde{v}}^{v}\left[L_{1} \int_{\widetilde{v}}^{v_{1}}\left[L_{2} \int_{\widetilde{v}}^{v_{2}} \frac{L_{3} \psi d v_{3}}{f\left(x, y, v_{3}\right)}\right] \frac{d v_{2}}{f\left(x, y, v_{2}\right)}\right] \frac{d v_{1}}{f\left(x, y, v_{1}\right)}, \quad L_{3}=\partial_{x}+v_{3} \partial_{y} ;
\end{aligned}
$$

Перепишем формулы (6), введя следующие обозначения:

$$
I_{k} g=\int_{\widetilde{v}}^{v_{k}} \frac{g\left(x, y, v_{k+1}\right) d v_{k+1}}{f\left(x, y, v_{k+1}\right)}, \quad k=0,1, \ldots,
$$

где положим $v_{0}=v$. В этих обозначениях

$$
U(x, y, v, \widetilde{v}, \varepsilon)=\psi-\varepsilon I_{0}\left(L_{1} \psi\right)+\varepsilon^{2} I_{0}\left(L_{1} I_{1}\left(L_{2} \psi\right)\right)-\varepsilon^{3} I_{0}\left(L_{1} I_{1}\left(L_{2} I_{2}\left(L_{3} \psi\right)\right)\right)+\ldots
$$

Сходимость этого степенного ряда доказана в работе [5]. Таким образом, установлено существование в области $\Omega_{x y w}$ аналитических по малому параметру интегралов системы $(2)$. В данной 
работе будет рассмотрен случай, когда $a_{1}=b_{1}=1, a_{2}=b_{2}=a=b=0$ (задача Дирихле). Пусть $v=F(x, y)$ является корнем уравнения $f(x, y, v)=0$, аналитическим в области $\overline{\omega_{x y}}$, а $\bar{y}(x)-$ решением задачи Коши

$$
y^{\prime}=F(x, y), \quad y(0)=0,
$$

которое аналитично на отрезке $[0,1]$. Положив $\psi(x, y)$ равной $\varphi(x)$, аналитической на отрезке $[0,1]$ и такой, что $\varphi(0)=0$, а затем равной $y-\bar{y}(x)$, построим два независимых интеграла:

$$
\begin{aligned}
& U^{[1]}(x, y, v, \widetilde{v}, \varepsilon)=\varphi(x)-\varepsilon\left(I_{0} \varphi^{\prime}\right)+\varepsilon^{2} I_{0}\left(L_{1}\left(I_{1} \varphi^{\prime}\right)\right)-\varepsilon^{3} I_{0}\left(L_{1} I_{1}\left(L_{2}\left(I_{2} \varphi^{\prime}\right)\right)\right)+\ldots \\
& U^{[2]}(x, y, v, \widetilde{v}, \varepsilon)=y-\bar{y}(x)-\varepsilon I_{0}\left(v_{1}-\bar{y}^{\prime}\right)+\varepsilon^{2} I_{0}\left(L_{1} I_{1}\left(v_{2}-\bar{y}^{\prime}\right)\right)-\ldots
\end{aligned}
$$

\section{3. Псевдоголоморфные решения и их продолжения.}

Определение 1. Решение $y(x, \varepsilon)$ краевой задачи (1) называется псевдоголоморфным в точке $\varepsilon=0$, если существует функция $Y(x, \eta, \varepsilon)$, аналитическая по третьей переменной в точке $\varepsilon=0$ при каждом $x \in[0,1]$ и каждом $\eta$ из некоторого неограниченного множества $T$, и такая, что для некоторой функции $\varphi(x)$ выполняется равенство для

$$
y(x, \varepsilon)=Y(x, \varphi(x) / \varepsilon, \varepsilon), \quad \forall x \in[0,1],
$$

когда $\varepsilon$ принадлежит достаточно малой окрестности значения $\varepsilon=0$.

Сформулируем достаточные условия существования псевдоголоморфного решения у краевой задачи.

Теорема 1. Пусть аналитическая на отрезке $[0,1]$ функи, $\varphi(x)$ такова, что $\varphi(0)=0$, и уравнение

$$
\varphi^{\prime}(x) \int_{\widetilde{v}}^{v} \frac{d v_{1}}{f\left(x, \bar{y}(x), v_{1}\right)}=\varphi(x) / \varepsilon
$$

имеет решение вида

$$
v=V_{0}(x, \Psi(\varphi(x) / \varepsilon), \widetilde{v}),
$$

в котором $q=\Psi(\eta)$ - иелая функиия с асимптотическим значением, равным $p_{0}$, и функиия $V_{0}(x, q, \widetilde{v})$ является аналитической на параллелепипеде $\Pi_{0}=[0,1] \times Q \times G$, где $Q$ и $G$-отрез$\kappa и$, причем $Q$ содержит точки $\Psi(0)$ и $p_{0}$. Тогда решение $y(x, \varepsilon)$ краевой задачи (1) является псевдоголоморфным в точке $\varepsilon=0$.

Доказательство. Вначале необходимо доказать, что при любом $\widetilde{v} \in G$ на всем отрезке $[0,1]$ существует решение задачи Коши

$$
\varepsilon y^{\prime \prime}=f\left(x, y, y^{\prime}\right) ; \quad y(0, \varepsilon)=0, \quad y^{\prime}(0, \varepsilon)=\widetilde{v}
$$

при достаточно малом положительном $\varepsilon$. Сведем задачу (9) к начальной задаче для системы

$$
\left\{\begin{array}{l}
y^{\prime}=v, \\
\varepsilon v^{\prime}=f(x, y, v) ; \\
y(0, \varepsilon)=0, \\
v(0, \varepsilon)=\widetilde{v},
\end{array}\right.
$$

и запишем первые интегралы этой системы:

$$
\left\{\begin{array}{l}
\widetilde{U}^{[1]}(x, y, v, \widetilde{v}, \varepsilon)=\varphi(x) / \varepsilon \\
y=\bar{y}(x)+\varepsilon \widetilde{U}^{[2]}(x, y, v, \widetilde{v}, \varepsilon)
\end{array}\right.
$$

где

$$
\begin{aligned}
& \widetilde{U}^{[1]}=I_{0} \varphi^{\prime}-\varepsilon I_{0}\left(L_{1}\left(I_{1} \varphi^{\prime}\right)\right)+\varepsilon^{2} I_{0}\left(L_{1} I_{1}\left(L_{2}\left(I_{2} \varphi^{\prime}\right)\right)\right)-\ldots ; \\
& \widetilde{U}^{[2]}=I_{0}\left(v_{1}-\bar{y}^{\prime}\right)-\varepsilon I_{0}\left(L_{1} I_{1}\left(v_{2}-\bar{y}^{\prime}\right)\right)+\ldots
\end{aligned}
$$


Вычислим значения функции $\Psi$ от левой и правой частей первого из уравнений системы (11):

$$
\Psi\left(\widetilde{U}^{[1]}(x, y, v, \widetilde{v}, \varepsilon)\right)=\Psi(\varphi(x) / \varepsilon) .
$$

Обозначим правую часть через $q$ и в левой части выделим главный член. В итоге имеем следующую систему:

$$
\left\{\begin{array}{l}
\Psi\left(I_{0} \varphi^{\prime}\right)+\varepsilon \Phi(x, y, v, \widetilde{v}, \varepsilon)=q, \\
y=\bar{y}(x)+\varepsilon \widetilde{U}^{[2]}(x, y, v, \widetilde{v}, \varepsilon) .
\end{array}\right.
$$

Возьмем $p>p_{0}$ очень близким к $p_{0}$, предположим, что $p_{0}<\Psi(0)$ и построим параллелепипед $\Pi=[0,1] \times[p, \Psi(0)] \times G$. Поскольку $W_{0}(x, q, \widetilde{v})$ аналитична на замкнутом параллелепипеде $\Pi_{0}$, то оценка ее модуля не зависит от $p$. Нетрудно видеть, что для системы (12) выполняются все условия теоремы о неявной функции и при $\varepsilon=0$ :

$$
\left\{\begin{array}{l}
v=V_{0}(x, q, \widetilde{v}) \\
y=\bar{y}(x) .
\end{array}\right.
$$

Отсюда можно утверждать, что в некой окрестности $\sigma_{x q \widetilde{w}}$ каждой точки $(x, q, \widetilde{v}) \in \Pi$ существует решение

$$
\left\{\begin{array}{l}
v=V(x, q, \widetilde{v}, \varepsilon), \\
y=Y(x, q, \widetilde{v}, \varepsilon),
\end{array}\right.
$$

системы (12), аналитическое в некоторой окрестности значения $\varepsilon=0$. Выберем из покрытия $\left\{\sigma_{x q \widetilde{v}}\right\}$ параллелепипеда П конечное подпокрытие, тогда функции (13) будут аналитическими в наименьшей окрестности $|\varepsilon|<\varepsilon_{0}$, соответствующей этому подпокрытию. Обозначим через $\widetilde{\Pi}$ прямоугольник, являющийся проекцией П на плоскость переменных $(x, q)$. Если величина параметра $\varepsilon$ в уравнении (1) удовлетворяет неравенству $0<\varepsilon<\varepsilon_{0}$, и кривая $\Gamma$, описываемая уравнением $q=\Psi(\varphi(x) / \varepsilon)$, целиком принадлежит $\widetilde{\Pi}$, то решение $(y(x, \varepsilon), v(x, \varepsilon))$ системы $(10)$ представимо в виде рядов:

$$
\left\{\begin{array}{l}
y(x, \varepsilon)=\bar{y}(x)+\sum_{n=1}^{\infty} \varepsilon^{n} Y_{n}(x, \Psi(\varphi(x) / \varepsilon), \widetilde{v}), \\
w(x, \varepsilon)=\sum_{n=0}^{\infty} \varepsilon^{n} V_{n}(x, \Psi(\varphi(x) / \varepsilon), \widetilde{v})
\end{array}\right.
$$

равномерно сходящихся на отрезке $[0,1]$. В случае, если прямоугольнику П принадлежит только часть кривой $\Gamma$, соответствующая $x \in\left[0, x_{1}\right]\left(0<x_{1}<1\right)$, то ряды (14) сходятся равномерно лишь на $\left[0, x_{1}\right]$. Обозначим это решение через $\left(y^{[0]}(x, \varepsilon), v^{[0]}(x, \varepsilon)\right)$. В этом случае его нужно продолжить вправо, для чего применим алгоритм псевдоголоморфного продолжения. Решим следующую задачу Коши:

$$
\left\{\begin{array}{l}
\frac{d y^{[1]}}{d x}=v^{[1]} \\
\varepsilon \frac{d v^{[1]}}{d x}=f\left(x, y^{[1]}, v^{[1]}\right), \\
y^{[1]}\left(x_{1}, \varepsilon\right)=y^{[0]}\left(x_{1}, \varepsilon\right), \\
\left.v^{[1]}\left(x_{1}, \varepsilon\right)=v^{[0]}\left(x_{1}, \varepsilon\right)\right) .
\end{array}\right.
$$

Запишем систему, аналогичную системе (11) первых интегралов:

$$
\left\{\begin{array}{l}
\widetilde{U}^{[1]}\left(x, y^{[1]}, v^{[1]}, v\left(x_{1}, \varepsilon\right), \varepsilon\right)=\left(\varphi(x)-\varphi\left(x_{1}\right)\right) / \varepsilon \\
y=\bar{y}(x)+\varepsilon \widetilde{U}^{[2]}\left(x, y^{[1]}, v^{[1]}, v\left(x_{1}, \varepsilon\right), \varepsilon\right) .
\end{array}\right.
$$

Она задает неявно решение $\left(y^{[1]}(x, \varepsilon), v^{[1]}(x, \varepsilon)\right)$ системы $(16)$. В итоге решение $\left(y^{[0]}(x, \varepsilon), v^{[0]}(x, \varepsilon)\right)$ продолжится на некоторый отрезок $\left[x_{1}, x_{2}\right]$, причем псевдоголоморфным образом, и т. д. Не ограничивая общности, будем предполагать, что регуляризирующая функция $\varphi(x)$, описывающая погранслой, строго монотонно убывает на промежутке $[0,1]$ (например, в методе погранфункций $\varphi(x)=-x)$, а функция $\Psi$, напротив, на интервале $\left(p_{0}, \Psi(0)\right)$ строго возрастает (в большинстве 
случаев это экспонента) [1,7]. При продолжении с помощью систем, аналогичных (16), получим цепочку следующих равенств:

$$
\frac{\varphi\left(x_{1}\right)}{\varepsilon}=\frac{\varphi\left(x_{2}\right)-\varphi\left(x_{1}\right)}{\varepsilon}=\cdots=\frac{\varphi\left(x_{m}\right)-\varphi\left(x_{m-1}\right)}{\varepsilon}=\ldots, \quad m=2,3, \ldots, j .
$$

По формуле Лагранжа $\varphi^{\prime}\left(\widetilde{x_{m}}\right)\left(x_{m}-x_{m-1}\right)=\varphi\left(x_{1}\right)$, где $\widetilde{x}_{m} \in\left(x_{m-1}, x_{m}\right), m=\overline{2, j}$. Так как $\varphi(x)$ голоморфна на отрезке $[0,1]$, то $\left|\varphi^{\prime}(x)\right| \leqslant l \forall x \in[0,1]$ для некоторой константы $l$, а значит, $x_{m}-x_{m-1} \geqslant\left|\varphi\left(x_{1}\right)\right| / l$ при каждом $m \in\{1,2,3, \ldots, j\}$. Таким образом, точки $x=1$ можно достичь за конечное число шагов. В итоге решение $y^{[0]}(x, \varepsilon)$ будет продолжено на весь отрезок, а под решением начальной задачи (10) будем понимать совокупность элементов $\left(y^{[0]}(x, \varepsilon), y^{[1]}(x, \varepsilon), \ldots, y^{[j]}(x, \varepsilon)\right)$.

Поскольку каждый элемент зависит от $\widetilde{v}$, то

$$
\left.y^{[j]}(x, \varepsilon)=T^{[j]}\left(x,\left(\varphi(x)-\varphi\left(x_{j-1}\right)\right) / \varepsilon\right), \widetilde{v}\right),
$$

откуда вытекает уравнение для определения $\widetilde{v}$ :

$$
\left.T^{[j]}\left(1,\left(\varphi(1)-\varphi\left(x_{j-1}\right)\right) / \varepsilon\right), \widetilde{v}\right)=0 .
$$

Одно из найденных значений подставим в формулы для $y^{[m]}(x, \varepsilon)(\bar{m}=\overline{1, j})$ и получим псевдоголоморфное решение $y(x, \varepsilon)$ краевой задачи $(1)$. Теорема доказана.

Приведем пример нахождения $y^{[0]}(x, \varepsilon)$ первого порядка, удовлетворяющего краевым условиям. Рассмотрим краевую задачу

$$
\varepsilon y^{\prime \prime}=x^{2}+y^{2} / 4+\left(y^{\prime}\right)^{2}-4, \quad x \in[0,1], \quad y(0, \varepsilon)=y(1, \varepsilon)=0 .
$$

Здесь предельная задача

$$
y^{\prime}=\sqrt{4-x^{2}-y^{2} / 4}, \quad x \in[0,1], \quad y(0)=0 .
$$

Докажем существование решения этой задачи на всем отрезке с помощью теоремы Чаплыгина [2]. Поскольку $0 \leqslant y^{\prime}(x) \leqslant 2$, то $0 \leqslant y(x) \leqslant 2$. Далее, $\tilde{f}(x, y)=\sqrt{4-x^{2}-y^{2} / 4}$ аналитична вместе со своей частной производной по $y$ на прямоугольнике $[0,1] \times[0,2]$, и легко построить нижнее и верхнее решения: $\bar{y}_{\mathrm{H}}=2 \sqrt{3} \sin (x / 2), \bar{y}_{\mathrm{B}}=4 \sin (x / 2)$. Значит, решение $\bar{y}(x)$ существует на всем промежутке. Вернемся к исходной задаче и сведем ее к системе:

$$
\left\{\begin{array}{l}
y^{\prime}=v \\
\varepsilon v^{\prime}=x^{2}+y^{2} / 4+v^{2}-4 \\
y(0, \varepsilon)=y(1, \varepsilon)=0
\end{array}\right.
$$

Здесь поверхностью $\Lambda$ служит эллипсоид $x^{2}+y^{2} / 4+v^{2}=4$. Будем строить интегралы этой системы в области $\Omega_{x y v}=\left\{(x, y, v): x^{2}+y^{2} / 4+v^{2}<4\right\}$, в которой $f(x, y, v)<0$. В результате, применив метод голоморфной регуляризации, получим решение $y_{1}^{[0]}(x, \varepsilon)$ первого порядка поставленной краевой задачи:

$$
y_{1}^{[0]}(x, \varepsilon)=\bar{y}(x)+\varepsilon \ln \frac{2 A(x)}{\widetilde{v}+A(x)-(\widetilde{v}-A(x)) e^{2 \bar{y}(x) / \varepsilon}},
$$

где

$$
A(x)=\sqrt{4-x^{2}-\bar{y}^{2}(x) / 4}, \widetilde{v}=A(1) \operatorname{th}(\bar{y}(1) / 2 \varepsilon) .
$$

В заключение следует отметить, что развитие метода голоморфной регуляризации может существенно оптимизировать решение нелинейных сингулярно возмущенных задач, так как данный метод применим также для уравнений и систем уравнений высших порядков. 


\section{СПИСОК ЛИТЕРАТУРЫ}

1. Васильева А. Б., Бутузов В. Ф. Асимптотические методы в теории сингулярных возмущений. - М.: Высшая школа, 1990.

2. Васильева А. Б., Нефедов Н. Н. Теоремы сравнения. Метод дифференциальных неравенств Чаплыгина. - М.: Изд-во МГУ, 2007.

3. Качалов В. И. Голоморфная регуляризация сингулярно возмущенных задач// Вестн. МЭИ/ - 2010. - 6. - C. $54-62$.

4. Качалов В. И. О голоморфной регуляризации сингулярно возмущенных систем дифференциальных уравнений// Ж. вычисл. мат. мат. физ. -2017 . -57 , № 4. - С. $64-71$.

5. Качалов В. И. Об одном методе решения сингулярно возмущенных систем тихоновского типа// Изв. вузов. Мат. $-2018 .-6$. - С. $25-30$.

6. Качалов В. И., Ломов С. А. Псевдоаналитические решения сингулярно возмущенных задач// Докл. PAH. - 1994. - 334, № 6. - C. 694-695.

7. Ломов С. А. Введение в общую теорию сингулярных возмущений. - М.: Наука, 1981.

8. Ломов С. А., Ломов И. С. Основы математической теории пограничного слоя. - М.: Изд-во МГУ, 2011.

9. Chang K. W, Howes F. A Nonlinear singular perturbation phenomena: theory and applications. - New York: Springer-Verlag, 1984.

Бесова Маргарита Ильинична

Национальный исследовательский университет «МЭИ», Москва

E-mail: besova.margarita@yandex.ru 